\title{
Systemic ALK-positive anaplastic large cell lymphoma with bilateral optic neurolymphomatosis resulting in permanent blindness
}

\author{
Karan Seegobin ${ }^{1}$, Muhamad Alhaj Moustafa ${ }^{1}$, Deborah Fischer ${ }^{1}$, Katelyn Keller ${ }^{1}$, \\ Jacquelyn Hastings ${ }^{1}$, Mohamed Kharfan Dabaja ${ }^{1}$, Ernesto Ayala ${ }^{1}$, Han W. Tun ${ }^{1}$, and \\ Liuyan Jiang ${ }^{1}$ \\ ${ }^{1}$ Mayo Clinic Hospital Jacksonville
}

July 7,2020

\begin{abstract}
A 26-year-old female developed bilateral blindness after a complete remission for ALK-positive ALCL. Initial workup showed neurolymphomatosis involving the bilateral optic nerves. She underwent hematopoietic cell transplantation and she remained in complete remission after 1 year; however her vision never improved. ALCL likely led to destruction of the optic pathway.
\end{abstract}

\section{Keywords}

ALK+ Anaplastic Large Cell Lymphoma; Neurolymphomatosis; Optic nerves

\section{Key Clinical Message}

ALK-positive ALCL can lead to rapid irreversible destruction of the optic pathway. Early treatment should be instituted in patients with CNS involvement or those at increased CNS risk.

\section{Introduction}

Neurolymphomatosis is a rare clinical entity in which lymphoma cells with neurotropism infiltrate and destroy peripheral nerves, spinal nerve roots, nerve plexuses and cranial nerves. [1,2,3] Neurolymphomatosis associated with $\mathrm{T}$ cell lymphoma is very rare.[1] In large series, $\mathrm{T}$ cell lymphoma was diagnosed in 2.5-10 \% of neurolymphomatosis cases $[1,2,3]$. We hereby report a very unusual case of CNS involvement by ALCL with bilateral optic neurolymphomatosis resulting in permanent blindness.

\section{Case}

A 26-year-old female was diagnosed about a year ago with stage IIA ALK+ ALCL with right axillary and cervical lymphadenopathy and was initiated on CHOEP (cyclophosphamide, doxorubicin, etoposide, vincristine and prednisone) chemotherapy. She achieved complete remission following three cycles of treatment. Soon thereafter, she developed sudden loss of vision in both eyes associated with fever, and night sweats. Vision loss started in the left eye then progressed to the right eye, with severity at a loss of light perception level. She was transferred to our institution. Significant clinical findings include loss of vision bilaterally with limited ability to appreciate light and dark stimulus. Pupils were dilated bilaterally both with a positive direct and indirect light reflex. She had left ocular dysmotility consistent with CN6 palsy. No focal weakness or sensory deficit bilaterally in the face and extremities. She had no lymphadenopathy, or hepatosplenomegaly. Serum lactate dehydrogenase enzyme level was elevated at $406 \mathrm{U} / \mathrm{L}$ (122-222U/L). MRI brain imaging showed bilateral nodular leptomeningeal enhancements in the posterior fossa and enhancement around both optic nerves 
(Fig.1 Before treatment) consistent with neurolymphomatosis. CSF cytology was positive for atypical large cells consistent with ALCL (Fig.2). Bone marrow biopsy did not show any involvement with lymphoma. She did not have any clinical evidence of neurolymphomatosis involving peripheral nerves. Review of her outside right axillary lymph node biopsy (Fig.3) confirmed the diagnosis of ALCL with the proliferation of large, atypical lymphocytes with horseshoe-shaped nuclei on hematoxylin and eosin stain and strong expression of ALK-1, CD5, and CD30, and negative expression of PAX 5, CD20, or CD3 by immunohistochemistry studies.

She was initially treated with dexamethasone $40 \mathrm{mg} /$ day for four days followed by a taper and the Ferrari regimen with high-dose methotrexate $3500 \mathrm{mg} / \mathrm{m} 2$ and high-dose cytarabine $2000 \mathrm{mg} / \mathrm{m} 2$ with intrathecal methotrexate $12.5 \mathrm{mg}$. This was followed with10 sessions of radiation to the bilateral orbits and optic nerves, a total cumulative dose of 3000cGy. There was no significant improvement in her vision after the initial treatment. She continued with the Ferrari regimen every 3 weeks for a total of 4 cycles. After 2 cycles there was no radiological evidence of residual lymphoma on follow up brain MRI imaging (Fig.1 Post treatment). Furthermore, repeat CSF cytology was negative for malignant cells. The patient subsequently underwent high-dose chemotherapy with BCNU and thiotepa followed by autologous stem cell transplant. One year after her transplant, she continues to be in complete remission for both systemic and CNS disease, and had small improvement in light and colour perception. In spite of her CNS disease being responsive to treatment and achieving complete remission her vision never improved significantly.

\section{Discussion}

Our patient has a very unique clinical presentation with bilateral optic neurolymphomatosis in the setting of treatment sensitive systemic ALK+ ALCL. Although she was treated promptly with radiographic complete response of CNS disease, her vision never recovered resulting in permanent blindness. It is understandable that the optic nerves and tracts were infiltrated and destroyed rather quickly and irreversibly. Our case appears to be the first case of bilateral optic neurolymphomatosis with ALCL reported in the literature as our extensive search did not turn up any such case.

Neurolymphomatosis of cranial nerves by T cell lymphoma is rare. [1] In a large study reported by International Primary CNS Lymphoma Collaborative Group (IPCG), the involvement of cranial nerves was seen in $46 \%$ of 50 patients.[1] In another large study from Massachusetts General Hospital (MGH), cranial nerves were involved in $51 \%$ of 72 patients. [2] However, a report from Mayo Clinic showed $10 \%(4 / 40)$ cranial nerve involvement. [3] Histologically, most cases of neurolymphomatosis are related to B cell lymphomas at $82 \%$ of cases (IPCG and MGH), [1, 2] and $97.5 \%$ of cases (Mayo). [3] T cell lymphomas account for $10 \%$ of cases (5/46 cases, IPCG study) and $5 \%$ of cases (4/72 cases, MGH study), and $2.5 \%$ of cases (1/40 cases, Mayo study). [1,2,3] Interestingly, one case series reported neurolymphomatosis of cranial nerves with leptomeningeal involvement in 4/5 patients with primary $\mathrm{T}$ cell CNS lymphoma. [4] Our patient had a similar distribution of CNS involvement.

In our patient, the diagnosis of optic neurolymphomatosis was supported by diffuse bilateral enhancement of optic nerves and tracts on MRI together with positive CSF cytology and known systemic ALK+ ALCL based on right axillary lymph node biopsy. We suggest that enhancement of cranial nerves on MRI with evidence of lymphoma in CSF or peripheral sites should be regarded as diagnostic of cranial neurolymphomatosis. The diagnosis can be quite difficult requiring meningeal and nerve biopsies when CSF studies and peripheral site biopsies are negative. $[1,2,3]$.

Primary or secondary CNS involvement of ALK+ ALCL is not common. [5] In a retrospective analysis of 600 patients with PTCL, the 5-year cumulative incidence of CNS relapse in ALK+ALCL is 5.4\% (4/74 patients). [1,2,3] There has been no standardized protocol to assess the CNS risk in patients with peripheral $\mathrm{T}$ cell lymphoma. Involvement of more than one extra nodal site is a significant risk factor for CNS relapse with bone, subcutaneous tissue, spleen, skin, lung, and liver regarded as high-risk sites. [6] Bone marrow involvement was reportedly associated with higher risk of CNS involvement in some reports. [7] Extra nodal involvement has a one-year cumulative incidence of $17 \%$ for CNS relapse. [6,8] Other risk factors for 
CNS relapse include elevated serum lactate dehydrogenase (LDH), and high International Prognostic Index (IPI) score $>/=3$, B symptoms, ALK+ histologic type and stage III-IV disease. [6,8] CNS prophylaxis for PTCL is controversial with little data reported. [6] Some authors advocate for evaluation of CNS at the time of diagnosis and possible CNS-directed prophylaxis in patients with ALK positivity and extra nodal involvement. [6] In our patient, the presence of ALK positivity, elevated LDH and B symptoms may have increased her risk for CNS relapse although she did not have extranodal involvement on initial diagnosis.

Interestingly she developed CNS disease after achieving complete response of systemic disease to three cycles of CHOEP chemotherapy. It is possible that CHOEP chemotherapy did not have prophylactic or therapeutic impact on CNS disease, or the damage may have already occurred. It is not surprising as chemotherapeutic agents in the regimen are known for poor CNS penetration. However, she had complete CNS response to CNS-penetrating agents, high-dose methotrexate and high-dose Ara-c. We recommend that CNS-penetrating agents should be used as early as possible in PTCL patients with high CNS risk or CNS disease. High-dose methotrexate-based treatment has been associated with improved survival in neurolymphomatosis and primary CNS T cell lymphoma. [3,9]

In conclusion, our case highlights devastating consequences secondary to CNS involvement by ALK+ ALCL. Novel biomarkers and clinical markers need to be identified for better CNS risk assessment in patients with ALCL. Early treatment with high-dose methotrexate-based regimen should be instituted in patients with CNS involvement or those at an increased CNS risk.

\section{Authorship contributions}

Karan Seegobin: Have made substantial contributions to conception and design, acquisition of data, and drafting the manuscript.

Muhamad Alhaj Moustafa: Have made substantial contributions to acquisition of data and drafting the manuscript.

Deborah Fischer: Have made substantial contributions to conception and design, acquisition of data, and drafting the manuscript.

Katelyn Keller: Have made substantial contributions to conception and design, acquisition of data, and drafting the manuscript.

Jacquelyn Hastings: Have made substantial contributions to conception and design, acquisition of data, and drafting the manuscript.

Mohamed A. Kharfan-Dabaja: Have made substantial contributions to conception and design, and revising it critically for important intellectual content.

Ernesto Ayala: Have made substantial contributions to conception and design, and revising it critically for important intellectual content.

Han W Tun: Have made substantial contributions to conception and design, and revising it critically for important intellectual content.

Liuyan Jiang: Have made substantial contributions to conception and design, and revising it critically for important intellectual content.

Conflict of interest

Mohamed A. Kharfan-Dabaja: reports consultancy for Pharmacyclics and Daiichi Sankyo

All other authors have no conflict of interest to declare

References 
1. Grisariu S, Avni B, Batchelor TT, et al. Neurolymphomatosis: International Primary CNS Lymphoma Collaborative Group report. International Primary CNS Lymphoma Collaborative Group Report. Blood. 2010; 115:5005-11.

2. Baehring JM, Damek D, Martin EC, et al. Neurolymphomatosis. Neuro Oncol. 2003; 5:104-115.

3. Novo M, Nowakowski GS, Micallef IN, et al. Clinical features, treatment and outcome of neurolymphomatosis: single institution experience. Hematological Oncology 2019. https://doi.org/10.1002/hon.80_2630

4. Levin N, Soffer D, Grissaru S, et al. Primary T-cell CNS lymphoma presenting with leptomeningeal spread and neurolymphomatosis. J Neurooncol. 2008; 90:77-83.

5. Wang CX, Wang H, Li J, et al. Brain metastasis of ALK positive anaplastic large cell lymphoma after a long-term disease-free survival in an old adult. Int J Clin Exp Pathol . 2014; 7:1182-1187.

6. Chihara D, Fanale MA, Miranda RN, et al. The risk of central nervous system relapses in patients with peripheral T-cell lymphoma.PLoS One . 2018; 13: e0191461.

7. Kuntegowdenahalli LC, Jacob LA, Komaranchath AS, Amirtham U. A rare case of primary anaplastic large cell lymphoma of the central nervous system. J Cancer Res Ther. 2015; 11:943-5.

8. Gurion R, Mehta N, Migliacci JC, et al. Central nervous system involvement in T-cell lymphoma: A single center experience. Acta Oncol . 2016; 55:561-566.

9. Shenkier TN, Blay JY, O'Neill BP, et al. Primary CNS Lymphoma of T-Cell Origin: A Descriptive Analysis From the International Primary CNS Lymphoma Collaborative Group. J Clin Oncol 2005;23:2233-2239

Figure1. MRI brain axial and sagittal sections show bilateral nodular leptomeningeal enhancements in the posterior fossa and enhancement around both optic nerves (Before treatment). MRI after 2 cycles show no radiological evidence of residual lymphoma (After treatment). 

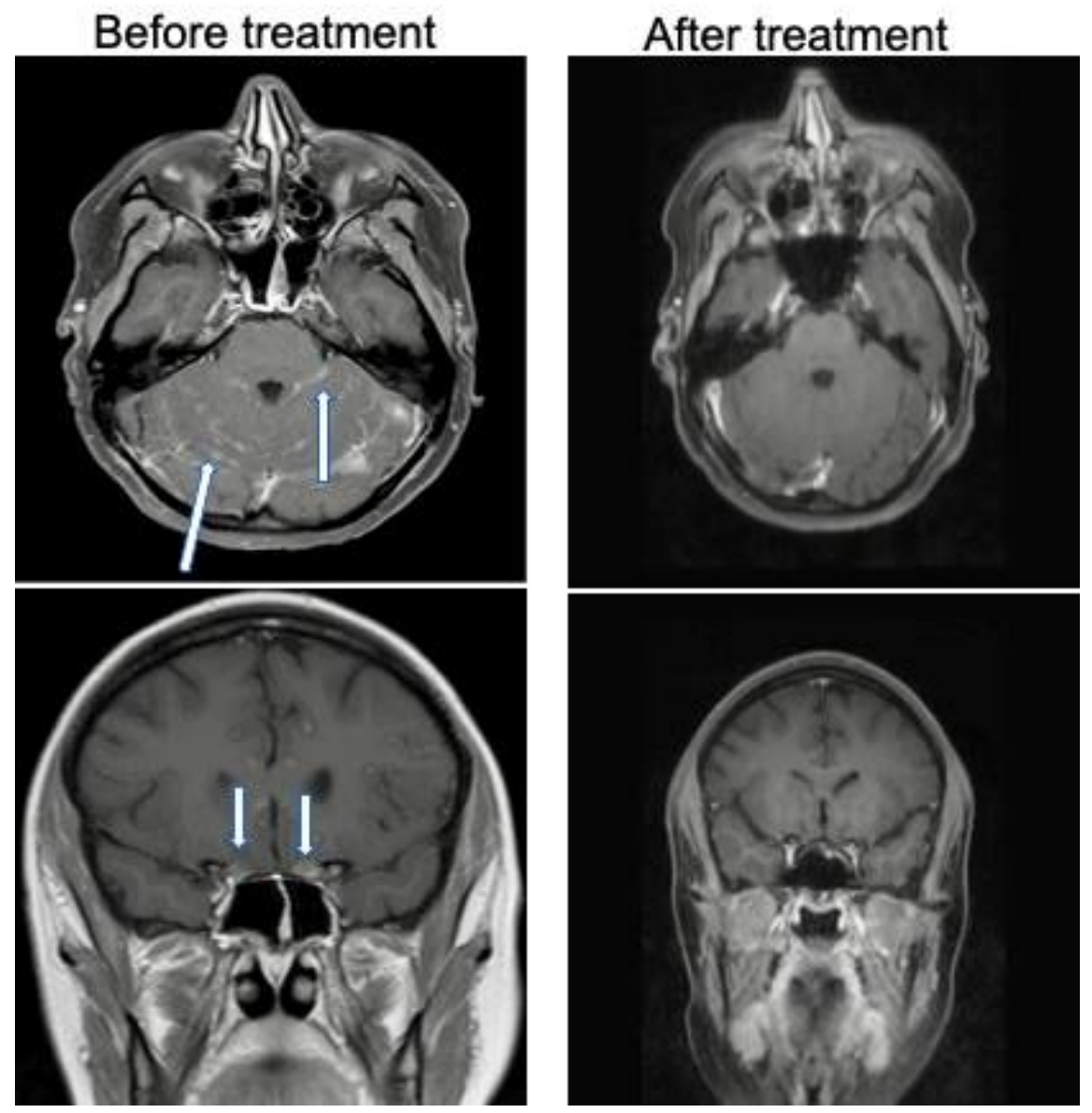

Figure 2. The cytospin slide from CSF fluid (modified Giemsa stain x20) contains many large atypical cells (see green arrow heads); and occasional mitosis is present (see black arrow)

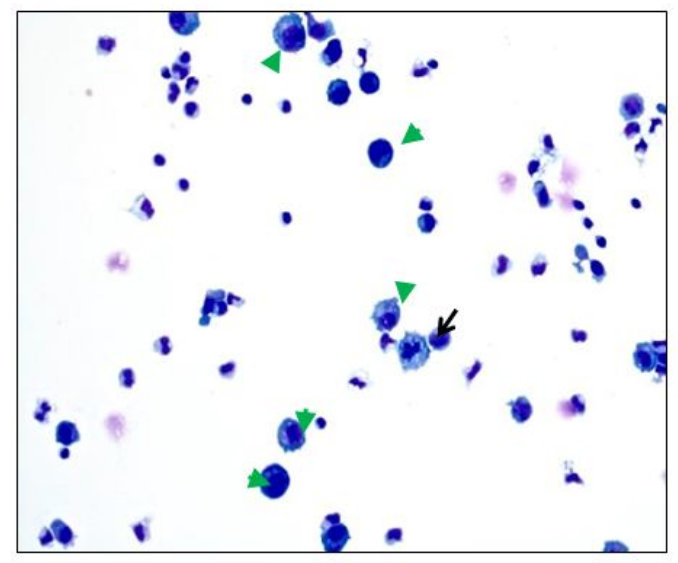

Figure 2: The cytospin slide from CSF fluid (modified Giemsa stain $\times 20$ ) contains many large atypical cells (see green arrow heads); and occasional mitosis is present (see black arrow). 
Figure 3. The $\mathrm{H}$ and $\mathrm{E}$ section of right axillary lymph node core needle biopsy (A, x20) show the numerous large atypical cells with horse-shoe shaped nuclei and prominent nucleoli. Immunohistochemistry studies show the neoplastic lymphocytes positive for CD 30 (B, x20), ALK-1 (C, x20), and CD 5 (D, x20); negative for PAX5, CD20, CD3.

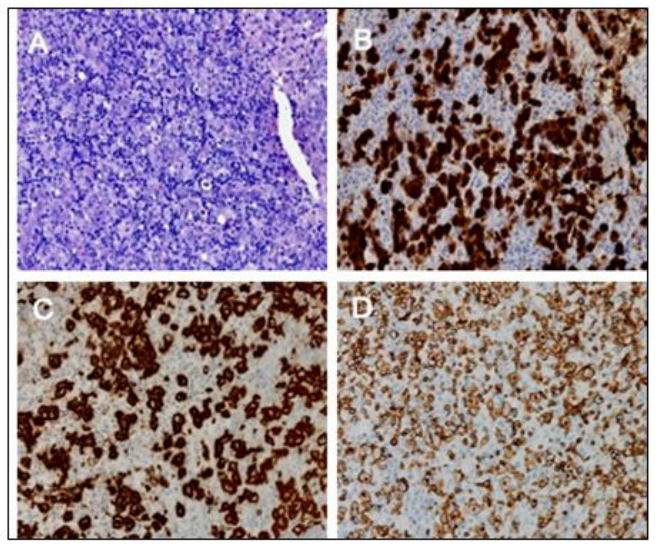

Figure 3: The H\&E section of right axillary lymph node core needle biopsy $(A, \times 20)$ show the numerous large atypical cells with horse-shoe shaped nuclei and prominent nucleoli. Immunohistochemistry studies show the neoplastic lymphocytes positive for CD30 (B, x20), ALK-1 (C, $\times 20)$, and CD5 (D, x20); negative for PAX-5, CD20, or CD3. 

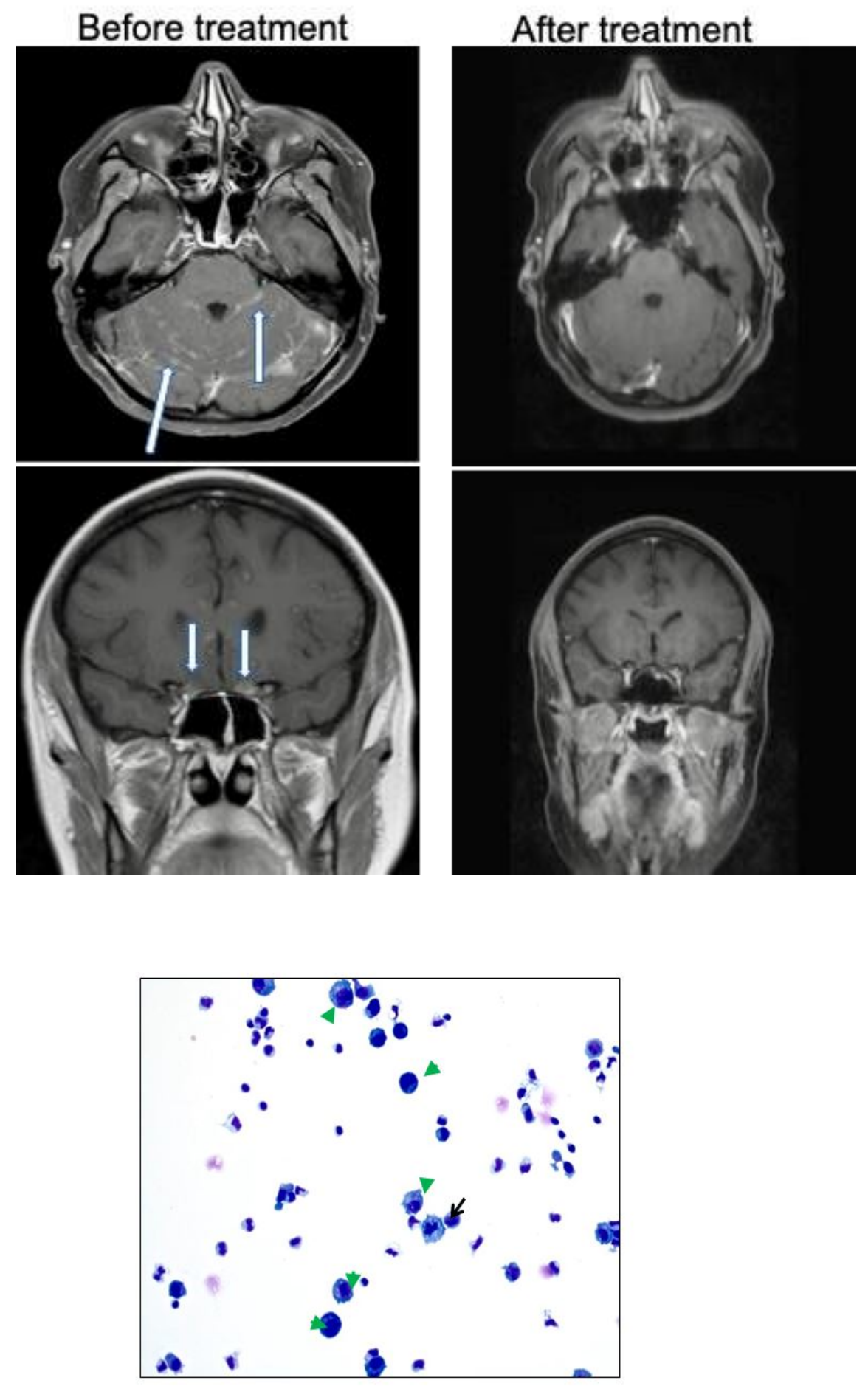

Figure 2: The cytospin slide from CSF fluid (modified Giemsa stain x20) contains many large atypical cells (see green arrow heads); and occasional mitosis is present (see black arrow). 


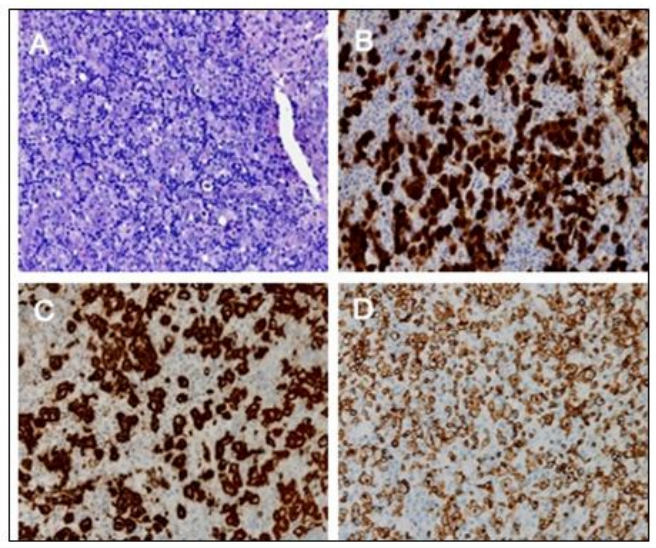

Figure 3: The H\&E section of right axillary lymph node core needle biopsy $(A, \times 20)$ show the numerous large atypical cells with horse-shoe shaped nuclei and prominent nucleoli. Immunohistochemistry studies show the neoplastic lymphocytes positive for CD30 (B, x20), ALK-1 (C, x20), and CD5 (D, x20); negative for PAX-5, CD20, or CD3. 